\title{
Contact Vitiligo Following Allergic Contact Dermatitis
}
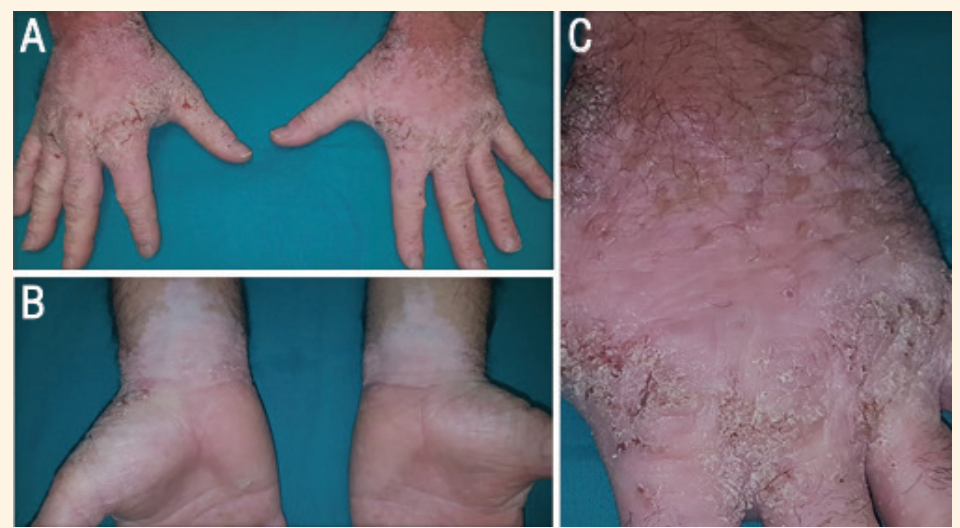

Figure 1: Photographs of the hands of a 45-year-old male patient showing (A) hyperkeratotic and fissured eczema on the back of both hands with skin depigmentation, (B) depigmentation on the ventral side of both wrists and (C) depigmentation of well-defined edges on the back of the hand.

A 45-YEAR-OLD MALE PATIENT WHO WAS A construction worker, with no personal history of psoriasis, atopic dermatitis or vitiligo, was referred to the Contact Eczema Department of a tertiary care hospital in Granada, Spain with chronic hand eczema and skin depigmentation over a period of 12 months. Regarding the primary eczema, skin depigmentation appeared a few months later. The patient reported the use of rubber gloves for many years. He had noticed itching and mild erythema over both hands. Currently, he wears nitrile gloves at work. Physical examination showed symmetric erythematous-squamous, hyperkeratotic and fissured plaques on both hands and ventral aspect of wrists [Figures 1A and 1B]. Skin depigmentation areas showed irregular edges [Figure 1C]. Wood's lamp examination accentuated that the depigmentation areas overlapped the eczema without vitiligo pattern [Figure 2]. No other anatomical sites were involved. Blood test results showed no significant alterations including tests from autoimmune thyroiditis, celiac disease and pernicious anaemia. Patch tests were performed with the European Comprehensive Baseline Series (Chemotechnique Diagnostics, Vellinge, Sweden), Rubber Additives Series (Chemotechnique Diagnostics) and hydroquinone monobenzylether 1\% pet (Shoe Series; Chemotechnique Diagnosis). The results were interpreted according to the criteria of the International Contact Dermatitis Research Group. Patch tests were read on day two and day four. The patient showed a strong positive patch test reaction to mercaptobenzothiazole (MBT). The patch test was performed with a piece of glove that the patient previously used which showed a positive reaction on day two and day four. Orthokeratotic epidermis of slightly reduced thickness with a conserved ridge pattern with hypopigmentation as well as a marked decrease in the number of melanocytes was observed in the histological examination [Figure 3A]. Melan-A, S100 and HMB-45 staining were absent from the depigmented lesions [Figure 3B]. At three and six months follow-up, a slight depigmentation of the area where MBT was tested was observed. Contact vitiligo following allergic contact dermatitis caused by MBT was diagnosed. Treatment with UVB-narrowband phototherapy was indicated. Almost complete improvement of eczema and depigmented areas was observed after at the six-month follow-up.

\section{Comment}

Contact vitiligo is the term used to describe an acquired form of skin depigmentation caused by certain chemicals, mainly phenolic/catecholic 

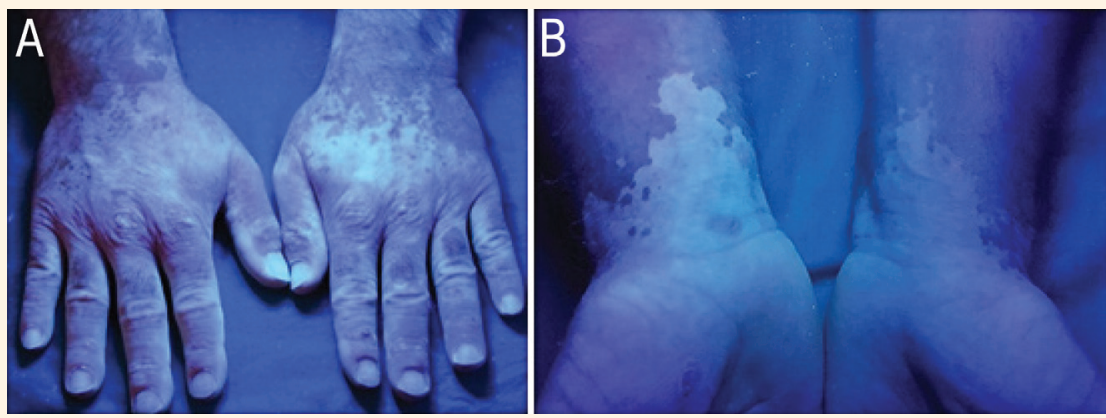

Figure 2: Wood's lamp examination photographs of the hands of a 45-year-old male patient showing enhancement of depigmented skin and no achromic areas, as is seen in vitiligo.

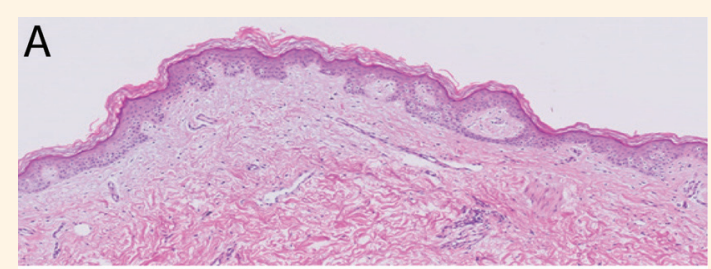

B

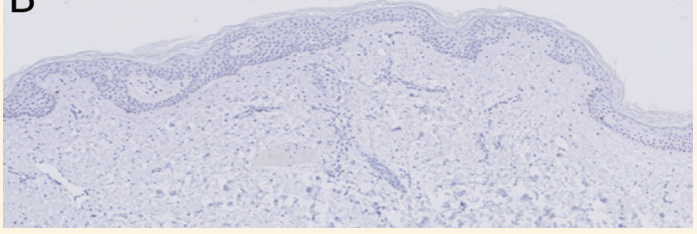

Figure 3: A: Haematoxylin and eosin staining showing orthokeratotic epidermis of slightly reduced thickness with a conserved ridge pattern with hypopigmentation. B: Immunostaining S100 protein at $\mathrm{x} 3$ magnification showing few melanocytes at both edges of the lesion.

derivatives. ${ }^{1}$ The terms contact leukoderma $(\mathrm{CL})$ and contact vitiligo $(\mathrm{CV})$, which have different meanings, may be confused in the literature. CL is the term used to describe depigmentation that develops after contact with chemicals or allergens, which may or may not be accompanied by allergic contact dermatitis. For a diagnosis of $\mathrm{CL}$, the patient must fulfil the following criteria: history of repeated exposure to depigmenting agent, must show numerous acquired confetti or pea-sized macules and the affected areas should correspond to the sites of chemical exposure. On the other hand, $\mathrm{CV}$ is an acquired leukoderma that occurs as a result of repeated topical or systemic exposure to a variety of chemicals, mainly alkyl phenols and catechols, regardless of the form of skin involvement. ${ }^{2}$ Ju et al. reported a case of contact vitiligo caused by IPPD. ${ }^{3}$ While CL is not frequent, and is clinically and histologically similar to vitiligo, it is sometimes confused with the latter. The first case was reported in 1939 and was caused by monobenzyl ether of hydroquinone in rubber gloves. ${ }^{4}$ Phenols and catechol derivatives are mainly responsible due to their direct toxic effect on melanocytes. ${ }^{5}$ Hydroquinone (monomethyl ether or monobenzyl ether), p-toluene diamine, monobenzone, azo dyes, aldehydes, epoxy resins, metals, dental acrylics, isopropanol or rotigotine, among others, have also been reported to lead to the development of contact leukoderma. ${ }^{6,7}$ MBT is used for natural and other rubber products as an accelerator, retarder and peptiser. This allergen can be found in items such as gloves, shoes, clothing, condoms, medical devices and adhesives.

\section{AUTHORS' CONTRIBUTION}

RR-V and FJN-T contributed equally to the design, drafting and critical review of the manuscript. Both authors approve the final version of the manuscript.

\section{References}

1. Boissy RE, Manga P. On the etiology of contact/occupational vitiligo. Pigment Cell Res 2004; 17:208-14. https://doi.org/10.1 111/j.1600-0749.2004.00130.x.

2. Harris JE. Chemical-induced vitiligo. Dermatol Clin 2017; 35:151-61. https://doi.org/10.1016/j.det.2016.11.006.

3. Ju HJ, Lee JH, Kim GM, Park CJ, Bae JM. Contact vitiligo induced by rubber ear loops from a disposable sanitary mask. Eur J Dermatol 2018; 28:690-1. https://doi.org/10.1684/ejd.2 018.3350 .

4. Oliver EA, Schwartz L, Warren LH. Occupational leukoderma: preliminary report. JAMA 1939; 113:927-8. https://doi. org/10.1001/jama.1939.72800350003010a.

5. Bonamonte D, Vestita M, Romita P, Filoni A, Foti C, Angelini G. Chemical leukoderma. Dermatitis 2016; 27:90-9. https://doi. org/10.1097/DER.0000000000000167.

6. Sinha S, Sardana K. Contact leukoderma following irritant contact dermatitis to an isopropanol-based hand rub: A consequence of rigorous hand hygiene. Contact Dermatitis 2021; 84:346-8. https://doi.org/10.1111/cod.13743.

7. Takeuchi A, Egawa G, Nomura T, Kabashima K. Contact leukoderma induced by rotigotine transdermal patch $\left(\right.$ Neupro $\left.^{\circ}\right)$. Eur J Dermatol 2019; 29:215-17. https://doi.org/10.1684/ejd.20 19.3500 . 